\section{PROGRESSIVE PATIENT CARE}

BY

\section{RONALD W. RAVEN, O.B.E., T.D., F.R.C.S.}

Surgeon, Royal Marsden Hospital, Westminster Hospital Teaching Group, and the French Hospital

Much has been written recently about the shortage of nurses. There has, however, been a considerable increase in the number of nurses employed in the hospital service since 1957. The Report of the Ministry of Health (1960) gives the following figures for wholetime nursing and midwifery staff (all grades): 147,676 in 1957 and 162,061 in 1960 . This increase, of course, has largely been offset by the reduction in weekly working hours from 48 to 44 recommended in 1957. In addition, since 1955 ward sisters and higher grades have had five instead of four weeks' annual leave, and recently it was announced that all third-year student nurses and higher grades (including State-enrolled nurses) in the hospital service shall have an extra week's leave. It is obvious, therefore, that in terms of available nursing hours there has been little increase over the past few years. The work, however, has undoubtedly increased, as shown by the rise in the numbers of in-patients and new out-patients, and by out-patient and casualty attendances, in addition to the growth of the different specialties.

As the number of nurses available is not sufficient to meet the increasing demands made upon them, it is obvious that nursing skills must be conserved, and the time has come for the medical profession to assist in using nursing services in the most effective way.

It is essential to relieve nurses of all non-nursing tasks that can be done by others. It would seem sensible to place highly trained nurses where their special skills are needed most, and utilize enrolled nurses, auxiliaries, and other grades for patients who do not require constant skilled nursing attention. In this country critically ill patients needing constant nursing care and the use of complicated and sometimes noisy equipment are still placed in beds adjacent to those occupied by ambulant or convalescent patients, who need an entirely different environment. In these conditions it is impossible to ensure that nursing skills are being used to advantage. A new grouping of patients is advocated, and medical staffs of hospitals should give this matter serious thought.

\section{Grouping of Patients}

Progressive patient care is the systematic grouping of patients according to their degree of illness and dependency on the nurse rather than by classification of disease and sex. Thus it is a method of planning the hospital facilities, both staff and equipment, to meet the individual requirements of the patient. In practice this means that those patients needing constant nursing attention and those who are ambulant and physically self-sufficient are removed from the ordinary ward unit and nursed in "intensive care units" and "self-care units" respectively. We should consider :

(1) Intensive care units for critically ill patients, regardless of diagnosis or sex, who need and would benefit from constant nursing attention. Skilled trained nurses would be assigned to these units and proper equipment provided for all the complicated nursing procedures now being used.
(2) Self-care units (or diagnosis or hostel beds) for convalescent patients or those requiring investigation. The facilities required in this unit are of the hotel or hostel type. An opportunity would be provided to teach the patients various health practices and give them instruction so that they could maintain their health on returning home.

(3) Intermediate care units for those patients not requiring to be housed in either of the foregoing, and who would constitute approximately $60 \%$ of all patients in hospital.

(4) Beds attached to out-patient departments for "oneday" patients. Many patients have minor operations under anaesthesia in the out-patient department and are able to return home after recovery. At present many of these cases are sent for recovery to ordinary hospital wards, thus interrupting and confusing the normal course of the nurses' work. Arrangements should be made to allocate a certain number of beds for out-patients.

\section{Concept of the System}

The title "progressive patient care" can give rise to misunderstanding, for it may be thought to mean the patient's "progress" through the various units in sequence, involving perhaps three or more moves during the average stay in hospital. This has been disproved in the U.S.A., where this system of nursing patients has been used in some hospitals for several years, since it has been found that few patients pass through three different sections. There is not much difficulty, therefore, in establishing a good patient/nurse relationship. It is admitted, however, that problems connected with the movements of patients have to be considered.

The principle of progressive patient care does not call for overnight wholesale rebuilding of hospitals but for a reorganization of the services. The concept can be applied equally well to one particular specialty or to one particular floor, and need not be applied to the entire hospital. Medical staff would feel more secure in that their seriously ill patients would be in a situation where constant medical and nursing attention was focused, and where the highest-skilled nurses were centred, with, in addition, all the equipment necessary for emergency treatment. Details of the various units are given below.

\section{Intensive Care Unit}

This unit must not be confused with the post-operative recovery room attached to the operating-theatre suite. It is designed for the acutely ill patient, regardless of diagnosis or sex, needing constant nursing attention throughout the 24 hours. The size of the unit varies according to whether it serves the entire hospital or only a section. In the former case it would comprise possibly about 10 to $12 \%$ of the total beds. Flexibility is provided by allocating some of the beds for intensive or intermediate care as required. The provision of one or two single-bed wards for cardiac, respiratory, or infectious cases is an advantage. It is important that the nurses have good visual control of all beds in the unit.

Type of Patient.-Any patient in a serious condition who has the slightest chance of recovery is placed in this unit, regardless of whether the condition is medical, surgical, infectious, psychiatric, orthopaedic, or gynaecological. Although deaths do occur in this unit, it is desirable not to admit patients here who are in the terminal stage of disease. The average length of stay would be from three to five days. 
Admission to the Unit.-A senior resident medical officer is selected to make the decisions for admission and discharge to and from the unit. Patients can be admitted from outside the hospital, or transferred from the casualty department, recovery room, or other wards. Doctors are responsible for the medical care of their individual patients. Every effort must be made to maintain full bed-occupancy and to make full use of the skilled nursing team. Relatives are allowed to visit the patients for short periods.

Equipment.-In the U.S.A. important features are the soundproofing and air-conditioning; a centrally placed nurses' station to facilitate observation of patients is essential. Sterile trays are laid ready for such procedures as tracheostomy, blood transfusion, lumbar puncture, etc. The beds should be $2 \frac{1}{2}$ to $3 \mathrm{ft}$. (76 to $91 \mathrm{~cm}$.) wide, fitted with adjustable sides; the head should be removable and the height easily changed. Piped oxygen and suction apparatus, oxygen tents, hypothermia equipment, and sphygmomanometers are also necessary.

\section{Intermediate Care Unit}

This unit is designed and staffed to care for patients whose nursing requirements are less than those available in the intensive unit, and would accommodate 60 to $70 \%$ of the total patients in the hospital. The services provided are similar to those in the usual medical or surgical ward. This unit admits ill patients and also patients transferred from the intensive unit. A large proportion of patients are admitted to and discharged from this unit without transfer to any other section of the hospital.

\section{Self-care Unit}

Patients admitted to this unit are ambulant and physically self-sufficient, and could well be attended by State-enrolled nurses or nursing auxiliaries under supervision of trained nurses. The services required, therefore, are chiefly supervisory or for health education. The unit is kept as simple and home-like as possible; no elaborate equipment is required, for nursing procedures are minimal. If possible, single rooms are provided with toilet facilities, and the unit can be established in a building detached from the main hospital, when covered ways should be provided. It should, however, be easily accessible. Patients may be admitted for diagnostic procedures, special treatment such as radiotherapy, or for preparation for major operations. They may be transferred from other sections of the hospital for convalescence. The nurse's role is not only that of support but also of teaching good health practicesworking closely with medical, catering, and other staffs. The transition between hospital and home is thus made easier for the patient.

This unit is urgently required in our hospitals, for it would prove of great benefit to patients, would save other hospital beds, and would free trained nurses for other work. Hospital and domiciliary organizations could be combined, and home nurses and health visitors could be introduced into the work which they would continue when the patient was discharged home.

\section{Recovery Unit}

This is not part of the "progressive patient care" organization, but it is mentioned here because it is essential to the modern hospital. The old method whereby patients were taken from the operating-theatres to the wards after various operations in different phases of anaesthesia had obvious disadvantages which have been overcome by the establishment of the recovery unit close to the operating-theatres. All patients are transferred there for variable periods according to their condition and requirements for the specialized postoperative medical and nursing care given by a combined medical and nursing team. Such a unit has been established at the Royal Marsden Hospital and has made an important contribution to the surgical work there. This unit is equipped with new adjustable beds, oxygen tent, piped oxygen, and suction apparatus to each bed. All the equipment of the intensive nursing care unit is available here. The nursing staff of the unit are specially trained in post-operative care. It is an obvious advantage to the surgeon to have easy access to his post-operative patients while he is still engaged in the operating-theatre.

\section{Conclusion}

Attention is called to a new organization of the beds of our hospitals on progressive nursing care principles. This organization ensures the efficient utilization of nurses, who will be in increasingly short supply in the future. Mention is also made of the recovery unit, which should be established in every hospital where major surgery is done. I realize that this paper may evoke criticism and hope that some discussion will follow.

\section{APPROVED NAMES}

The last supplement to the consolidated list of " approved names" was published in the Journal of November 11 (p. 1282). A new supplement, dated December, 1961, is printed below. Communications relating to "approved names" should be addressed to the Secretary, British Pharmacopoeia Commission, General Medical Council, 44 Hallam Street, London W.1.

\begin{tabular}{|c|c|c|}
\hline Approved Name & Other Names & Notes \\
\hline Carbinoxamine & $\begin{array}{l}\text { Polymeric condensation product of } \\
\text { aluminium oxide and O-acetylsali- } \\
\text { cylic acid, } \\
\text { "Palaprin" } \\
p \text {-Chloro- } \alpha-2 \text {-pyridylbenzyl 2-dimethyl- } \\
\text { aminoethyl ether } \\
\text { "Clistin" is the maleate }\end{array}$ & Antihistamine \\
\hline $\begin{array}{l}\text { Dichloralphen- } \\
\text { azone }\end{array}$ & $\begin{array}{l}\text { A complex of chloral hydrate and } \\
\text { "phenazone, }\end{array}$ & Sedative \\
\hline Dydrogesterone & 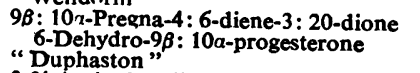 & Progestogen \\
\hline Etryptamine & $\begin{array}{l}\text { obutylindole } \\
\text { ptamine } \\
\text { "is the acetate }\end{array}$ & $\begin{array}{l}\text { Mono-amine } \\
\text { oxidase } \\
\text { inhibitor }\end{array}$ \\
\hline Fluocinolone $\ldots$ & $\begin{array}{l}\text { 6a: } 9 \alpha-D i f u o r o-11 \beta: 16 a: 17 \alpha: 21- \\
\text { tetrahydroxypregna-1:4-diene- } \\
\text { 3:20-dione } \\
6 \alpha: 9 a-D i f l u o r o-16 a-h y d r o x y p r e d n i s o- \\
\text { lone } \\
\text { "Synalar" is the acetonide }\end{array}$ & $\begin{array}{l}\text { Adrenocortical } \\
\text { steroid }\end{array}$ \\
\hline Methyl cyste & $\begin{array}{l}\text { Methyl } \alpha \text {-amino- } \beta \text {-mercaptopropionate } \\
\text { "Acdrile" is the hydrochloride }\end{array}$ & $\begin{array}{l}\text { Treatment of } \\
\text { chronic }\end{array}$ \\
\hline Pentalamide $\quad \ldots$ & $\begin{array}{l}\text { o-Pentyloxybenzamide } \\
\text { O-Pentylsalicylamide }\end{array}$ & $\begin{array}{l}\text { bronc } \\
\text { Fungici }\end{array}$ \\
\hline Phenaglycodol .. & $\begin{array}{l}\text { 2-p-Chlorophenyl-3-methylbutane- } \\
\text { " 3-diol } \\
\text { "Uitran" }\end{array}$ & Sedative \\
\hline Phenbenicillin .. & $\begin{array}{l}\text { a-Phenoxybenzylpenicillin } \\
\text { "Penspek" is the potassium salt }\end{array}$ & Antibiotic . \\
\hline Sodium metrizoate & 3-Acetamido-2: $4: 6$-tri-iodo-5- $N$ - & \\
\hline Thiethylperazine & $\begin{array}{l}\text { 2-Ethylthio-10-[3-(4-piperazin-1-yl)- } \\
\text { propyl]-phenothiazine } \\
\text { The name " torecan " is applied to the } \\
\text { base and its salts }\end{array}$ & $\begin{array}{l}\text { medium } \\
\text { Sedative and } \\
\text { antiemetic }\end{array}$ \\
\hline
\end{tabular}

